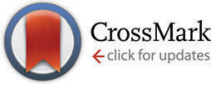

Cite this: Chem. Commun., 2015, 51,17128

Received 15th September 2015, Accepted 2nd October 2015

DOI: $10.1039 / c 5 c c 07745 a$

www.rsc.org/chemcomm

\section{Catalytic photoinduced electron transport across a lipid bilayer mediated by a membrane-soluble electron relay $\dagger$}

\author{
B. Limburg, E. Bouwman and S. Bonnet*
}

\begin{abstract}
Unidirectional photocatalytic electron transfer from a hydrophilic electron donor encapsulated in the interior of a liposome, to a hydrophilic electron acceptor on the other side of the membrane, has been achieved using the simple membrane-soluble electron relay 1-methoxy- $N$-methylphenazinium $\left(\mathrm{MMP}^{+}\right)$. The total amount of photoproduct ( $>140 \mathrm{nmol}$ ) exceeds the number of moles of $\mathrm{MMP}^{+}$present $(125 \mathrm{nmol})$, thus showing that the transport of electrons is catalytic.
\end{abstract}

To accomplish efficient artificial photosynthesis, two complimentary redox reactions must be fuelled by visible light irradiation. In both photoreactions the generation of a long-lived chargeseparated species is required, and the greatest challenge in photocatalysis is to overcome charge recombination. In order to do so, the reduced electron acceptor and the oxidized electron donor must be physically separated. In green plants for example such separation is realized by large protein molecules embedded in the thylakoid membrane of chloroplasts. The dissymmetry of biological membranes allows for unidirectional electron transfer to occur from the lumen to the stroma. However, such photocatalytically active protein assemblies are highly complex and fragile, and artificial systems based on simpler, more robust molecules are required for building artificial devices capable of solar fuel production.

Among the numerous tools offered by supramolecular photocatalysis liposomes holds great promise. ${ }^{1}$ They offer a simple way to organize molecular components in space and mimic biological photosynthesis. By segregating electron donors and acceptors on each side of a lipid membrane, it should be possible to create long-lived charge-separated states that, in combination with two different catalysts, would allow for producing solar fuels. ${ }^{1}$ Electron transfer across the liposomal membrane to

Gorlaeus Laboratories, Leiden Institute of Chemistry, Leiden University, P.O. Box 9502, 2300 RA, Leiden, Netherlands.E-mail: bonnet@chem.leidenuniv.nl $\dagger$ Electronic supplementary information (ESI) available: Photocatalytic results in the presence of $\mathrm{O}_{2}$ or in the absence of electron acceptor WST1 ${ }^{-}$. Detailed experimental procedures. See DOI: 10.1039/c5cc07745a electron acceptors such as quinones, ${ }^{2} \mathrm{Co}(\mathrm{bpy}){ }_{3}{ }^{3+}, 3$ or viologens ${ }^{4}$ has been investigated intensively in the past. However, especially with viologens some ambiguity about the true nature of the charge separation was later revealed. ${ }^{5}$ It was shown that in those systems electron transport occurred due to diffusion of the reduced electron acceptor through the membrane, after which it remained occluded in the interior of the liposome. Thus, because the electron-transporting molecule never returned to its original location, it was unable to turn over for producing a true catalytic reaction. Up to now, there are only few systems known in the literature that catalytically transport electrons across membranes. ${ }^{2 a, d-f, 6}$ However, all of them contain a complicated molecular structure to realize the charge separation across the membrane.

Herein, we report the truly catalytic unidirectional photoinduced electron transfer from the interior of a liposome to its exterior, based largely on commercially available compounds. This study is inspired by the widely used "WST-1" cell-counting assay, in which a water-soluble electron acceptor, the 2-(4iodophenyl)-3-(4-nitrophenyl)-5-(2,4-disulfophenyl)-2H-tetrazolium anion (WST1 ${ }^{-}$, Scheme 1), is reduced to the corresponding formazan dye $\left(\mathrm{Fz}^{2-}{ }^{2-}\right.$ Scheme 1) by addition of two electrons

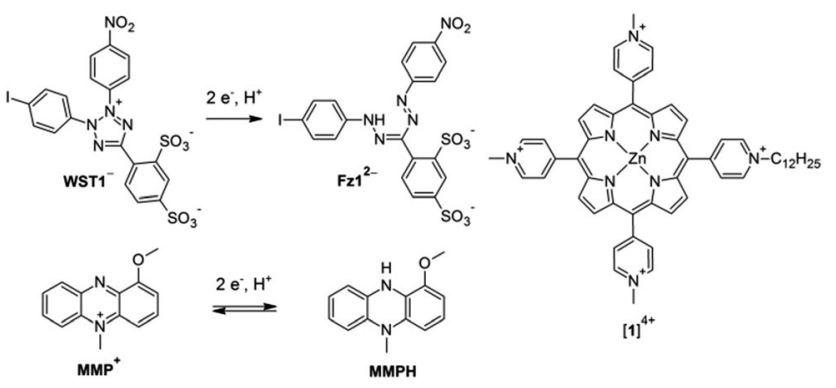

Scheme 1 Chemical structures of the membrane-insoluble electron acceptor 2-(4-iodophenyl)-3-(4-nitrophenyl)-5-(2,4-disulfophenyl)-2Htetrazolium anion (WST1 ${ }^{-}$), the membrane-soluble electron relay 1-methoxy$\mathrm{N}$-methylphenazinium cation $\left(\mathrm{MMP}^{+}\right)$, their reduction products $\mathrm{Fz}^{2-}$ and $\mathrm{MMPH}$, respectively, and the lipophilic zinc porphyrin photosensitizer $[\mathbf{1}]^{4+}$. 
and one proton. Usually the electrons and protons are generated inside living cells in the form of nicotinamide adenine dinucleotide (NADH), which is too hydrophilic to cross the cell membrane. The WST-1 assay requires the membrane-soluble relay 1-methoxy$N$-methylphenazinium cation $\left(\mathrm{MMP}^{+}\right.$, Scheme 1) to transport the biological reducing equivalents from inside the cell to the WST1 ${ }^{-}$ acceptor situated on the other side of the membrane. ${ }^{7}$ By analogy, we envisioned that biomimetic liposomes containing a watersoluble electron donor in their interior (ethylenediaminetetraacetic acid, $\mathrm{H}_{4}$ EDTA) and a photosensitizer in the membrane would be able to unidirectionally transport the photoelectrons to the $\mathrm{WST}^{-}$molecules located outside the liposomes, provided that $\mathrm{MMP}^{+}$is added to the system. ${ }^{7}$ We describe below photocatalytic studies showing that the lipid membrane diminishes product inhibition, while the use of membrane-insoluble electron donors and acceptors allows for avoiding the mechanistic ambiguities generally associated with this type of studies.

Dye-functionalized liposomes were prepared in which the amphiphilic photosensitizer $[\mathbf{1}]^{4+}$ (Scheme 1) was added in the membrane, the electron donor HEDTA ${ }^{3-}$ was placed in the inner water pool of the liposome, and the electron acceptor WST1 ${ }^{-}$was added into the bulk solution, i.e., on the outside of the liposome. The liposome bilayer composition consisted of 1,2-dipalmitoyl-sn-glycero-3-phosphocholine (DPPC), $1.6 \mathrm{~mol} \%$ of the alkyl-functionalized zinc porphyrin $[1] \mathrm{Cl}_{4}$, and $1 \mathrm{~mol} \%$ of the pegylated lipid sodium $N$-(carbonyl-methoxypolyethylene glycol-2000)-1,2-distearoyl-sn-glycero-3-phosphoethanolamine (NaDSPE-PEG2K) to stabilize the liposome dispersion. The liposomes were prepared by standard extrusion methods in an HEDTA $^{3-}$ buffer at $\mathrm{pH}=8$, and were subsequently purified by size-exclusion chromatography to remove non-encapsulated HEDTA $^{3-}$ (see ESI $\dagger$ ). To this liposomal solution (called hereafter liposome sample LA) was then added an isotonic solution containing the electron acceptor $\mathrm{WST}^{-}$(as the sodium salt), the electron relay $\mathrm{MMP}^{+}$(as methylsulfate salt), and $\mathrm{ZnSO}_{4}$. The addition of $\mathrm{Zn}^{2+}$ ions allowed for binding and thus inactivating traces of HEDTA ${ }^{3-}$ that may remain in the bulk aqueous phase after size-exclusion chromatography. ${ }^{4 g, h}$ The resulting mixtures, called system $\mathbf{L A}_{\mathbf{1}}$ or $\mathbf{L}_{\mathbf{2}} \mathbf{A}_{\mathbf{1}}$ (see compositions in Table 1), were de-aerated and irradiated in the Soret band of $[1]^{4+}\left(\lambda_{\text {irr }}=449 \mathrm{~nm}\right)$. The excited photosensitizer was assumed to not differ significantly from the well-characterized methyl analogue $[\mathrm{ZnTMPyP}]^{4+}$, i.e., to be of triplet character and to have an oxidation potential of $\sim+0.8 \mathrm{~V} v s$. NHE and a reduction potential of $\sim-0.45 \mathrm{~V} v s . \mathrm{NHE}^{8}$

A typical evolution of the UV-vis spectrum of the irradiated dispersions as a function of irradiation time is shown in Fig. 1a. Clearly, a band ascribed to the $\mathrm{Fz}^{2-}$ dye arises at $\lambda_{\max }=438 \mathrm{~nm}$ as a result of the photoinduced reduction of $\mathrm{WST}^{1-}{ }^{7}$ The amount of photoproduct, $n_{\mathrm{Fz} 1}$, determined by the absorbance due to $\mathrm{Fz}^{2-}\left(\varepsilon_{438}=37000 \mathrm{M}^{-1} \mathrm{~cm}^{-1}\right){ }^{7}$ was plotted as a function of the number of photons $Q(t)$ absorbed by system since $t=0$ (Fig. 1b, $\mathbf{L}_{\mathbf{2}} \mathbf{A}_{\mathbf{1}}$ ). The slope of this curve at $t=0$ was determined to be $1.2(1) \%$ in both $\mathbf{L A}_{\mathbf{1}}$ and $\mathbf{L}_{\mathbf{2}} \mathbf{A}_{\mathbf{1}}$. However, as only half of the photosensitizers are assumed to participate in the reaction, only half of the photons are effectively used. The quantum yield for $\mathrm{Fz}^{2-}$ formation, $\varphi_{0}$, is thus twice higher
Table 1 Bulk concentrations and initial quantum yields for the different photocatalytic liposomes described in this report

\begin{tabular}{lllllll}
\hline System $^{a}$ & $\begin{array}{l}\mathbf{M M P}^{+b} \\
(\mu \mathrm{M})\end{array}$ & $\begin{array}{l}\text { Triton X-100 } \\
(\mu \mathrm{M})\end{array}$ & $\mathrm{O}_{2}$ & $\begin{array}{l}\text { EDTA } \\
(\mathrm{mM})\end{array}$ & $\begin{array}{l}\mathrm{ZnSO}_{4} \\
(\mathrm{mM})\end{array}$ & $\begin{array}{l}\varphi_{0} \\
(\%)\end{array}$ \\
\hline $\mathbf{L A}_{\mathbf{1}}$ & 42 & - & - & $c$ & 5 & 2.4 \\
$\mathbf{L A}_{2}{ }^{2}$ & - & - & - & $c$ & 5 & - \\
$\mathbf{L}_{2} \mathbf{A}_{\mathbf{1}}{ }^{d}$ & 42 & - & - & $c$ & 5 & 2.4 \\
$\mathbf{L}_{2} \mathbf{A}_{2}{ }^{d}$ & - & - & - & $c$ & 5 & - \\
$\mathbf{L A}_{3}{ }{ }^{d}$ & 42 & 0.3 & - & $c$ & 5 & - \\
$\mathbf{L}_{2} \mathbf{A}_{4}{ }^{d}$ & 42 & - & Yes & $c$ & 5 & - \\
$\mathbf{L B}_{1}$ & 42 & - & - & 125 & - & 0.90 \\
$\mathbf{L B}_{2}$ & - & - & - & 125 & - & 0.72 \\
$\mathbf{L B}_{3}$ & - & 0.3 & - & 125 & - & 3.8 \\
$\mathbf{L B}_{\mathbf{4}}$ & - & - & Yes & 125 & - & 0.84
\end{tabular}

${ }^{a}$ For the details on the preparation of liposome samples, see ESI. Bulk concentrations before extrusion and/or size exclusion: DPPC (1.04 mM), NaDSPE-PEG2K $(10.4 \mu \mathrm{M}),[1] \mathrm{Cl}_{4}(16.7 \mu \mathrm{M})$. Final concentration of [1]Cl4 $\sim 2.1 \mu \mathrm{M}$, as determined by UV-vis. ${ }^{b} \mathrm{MMP}^{+}$added as methylsulfate salt. ${ }^{c}$ For these samples, $\mathrm{Na}_{3}$ HEDTA was encapsulated inside the liposomes. To obtain the same osmolality inside and outside the liposomes, all solutions were prepared using isotonic $\mathrm{NH}_{4} \mathrm{OAc}(379$ mOsm, $\mathrm{pH}=7)$. ${ }^{d}$ These samples contained twice more liposomes per unit volume than LA (DPPC: $2.08 \mathrm{mM}$, NaDSPE-PEG2K: $20.8 \mu \mathrm{M},[\mathbf{1}] \mathrm{Cl}_{4}: 33 \mu \mathrm{M}$ ).
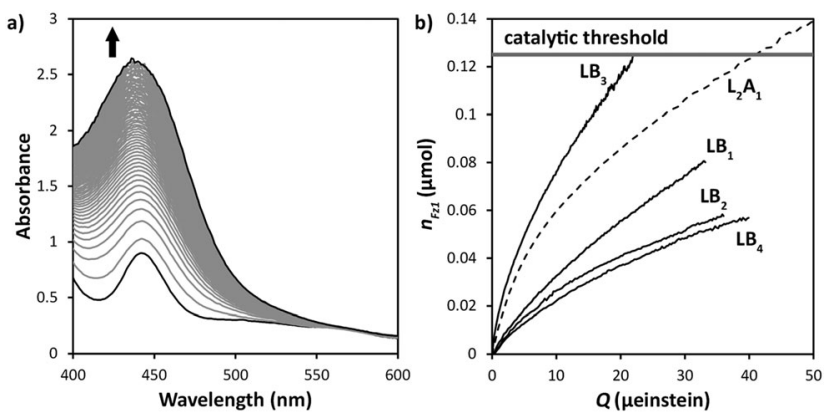

Fig. 1 (a) Evolution of the UV-vis spectrum of $\mathbf{L}_{2} \mathbf{A}_{1}$ (see Table 1) under blue light irradiation. Spectra were recorded every minute. (b) Photocatalytic formation of $\mathrm{Fz1}^{2-}\left(n_{\mathrm{Fz} 1}\right)$ as a function of the amount of photons $Q(t)$ absorbed by the porphyrin since $t=0$. See Table 1 for the composition of systems $\mathrm{L}_{2} \mathrm{~A}_{1}$ and $\mathrm{LB}_{1-4}$. Conditions: $T=298 \mathrm{~K}, \lambda_{\text {irr }}=449 \mathrm{~nm}, \Delta \lambda_{\frac{1}{2}}=25 \mathrm{~nm}$, photon flux $\Phi=3.3 \times 10^{-8}$ einstein $\mathrm{s}^{-1}$. Total irradiation time is 30 minutes, or shorter if the absorbance of the solution became higher than $A=3$ at $438 \mathrm{~nm}$.

(2.4(2)\%). Dynamic light scattering analysis of the mixture before and after $60 \mathrm{~min}$ irradiation gave essentially the same results $\left(Z_{\text {ave }}=166 \mathrm{~nm}\right.$, PDI $=0.13$ before; $Z_{\text {ave }}=162 \mathrm{~nm}$, PDI $=$ 0.16 after), indicating that the liposomes stayed intact during photocatalysis. Sample $\mathbf{L A}_{\mathbf{1}}$ is thus able to photocatalytically transport the reducing equivalents from inside the liposomes $\left(\mathrm{HEDTA}^{3-}\right.$ ) to the outside (WST1 ${ }^{-}$), thus realizing transmembrane charge separation. In $\mathbf{L}_{2} \mathbf{A}_{\mathbf{1}}$, the twice-higher liposome concentration allowed for observing that more $\mathrm{Fz}^{2-}$ was produced during the reaction (141 nmol after 1 hour of irradiation) than the number of moles of $\mathrm{MMP}^{+}$present $(125 \mathrm{nmol})$ in the system (Fig. 1b), indicating that $\mathrm{MMP}^{+}$functioned as a true catalyst in this system.

When the electron relay $\mathrm{MMP}^{+}$was omitted from $\mathbf{L A}_{\mathbf{1}}$ or $\mathbf{L}_{\mathbf{2}} \mathbf{A}_{\mathbf{1}}$ (systems $\mathbf{L A}_{\mathbf{2}}$ or $\mathbf{L}_{\mathbf{2}} \mathbf{A}_{\mathbf{2}}$, respectively, see Table 1), formation of $\mathrm{Fz}^{2-}$ was not observed. Instead the Soret band of the porphyrin bleached to around half of its initial intensity 
(Fig. S1a, ESI $\dagger$ ). It was thus clear that systems $\mathbf{L A}_{\mathbf{1}}$ and $\mathbf{L}_{\mathbf{2}} \mathbf{A}_{\mathbf{1}}$ require $\mathrm{MMP}^{+}$to perform charge separation, and that the electrons cannot be transferred directly from HEDTA $^{3-}$ to WST1 $^{-}$because they are positioned in two different aqueous phases separated by an impermeable lipid membrane. Furthermore, in $\mathbf{L} \mathbf{A}_{\mathbf{2}}$ and $\mathbf{L}_{\mathbf{2}} \mathbf{A}_{\mathbf{2}}$ only half the porphyrin molecules were bleached, probably those on the inside of the membrane that were photoreduced by $\mathrm{HEDTA}^{3-}$. In system $\mathbf{L A}_{3}$, triton X-100 was added to $\mathbf{L A}_{\mathbf{1}}$ prior to the reaction, resulting in disruption of the liposomes and release of $\mathrm{HEDTA}^{3-}$ into the bulk solution. In such conditions neither formation of $\mathrm{Fz}^{2-}$, nor bleaching of the porphyrin was observed upon light irradiation. Since the bulk concentration of HEDTA $^{3-}$ was $0.11 \mathrm{mM}$ (see $\mathrm{ESI} \dagger$ ), i.e. $\sim 50$ times lower than the bulk concentration of $\mathrm{Zn}^{2+}$, the released HEDTA ${ }^{3-}$ was fully bound to the $\mathrm{Zn}^{2+}$ ions thereby losing its ability to donate electrons in system $\mathbf{L A}_{\mathbf{3}}$.

In order to investigate in which way physical separation of the electron donor and acceptor by the lipid membrane influences the rate of the photocatalytic reaction, the phototcatalytic system $\mathbf{L B}_{\mathbf{1}}$ was developed, which contained both the electron donor and acceptor on the outside of the liposome membrane (Fig. 2, bottom). The liposomes used for these systems, called liposome sample LB, were prepared by standard extrusion methods from a lipid film of DPPC, 1 mol\% NaDSPEPEG2K and $1.6 \mathrm{~mol}^{2}[1] \mathrm{Cl}_{4}$ hydrated in aqueous $\mathrm{NH}_{4} \mathrm{OAc}(\mathrm{pH}=$ 7.0). To this liposomal solution were added an isotonic HEDTA $^{3-}$ buffer at $\mathrm{pH}=8.0, \mathrm{WST}^{-}$, and the electron relay $\mathrm{MMP}^{+}$, to form system $\mathbf{L B}_{\mathbf{1}}$ (see Table 1). System $\mathbf{L B}_{\mathbf{1}}$ was deaerated and irradiated as described above, which also led to the formation of $\mathrm{Fz}^{2-}$. A much lower initial photoelectron transfer quantum yield $\varphi_{0}=0.90(9) \%$ was found for this system compared to $\mathbf{L} \mathbf{A}_{\mathbf{1}}$, indicating that the physical separation of the electron donor and acceptor in $\mathbf{L A}_{\mathbf{1}}$ leads to a more stable separation of charges (Fig. 1b). In system $\mathbf{L B}_{\mathbf{1}}$, the photoreduced electron acceptor $\mathrm{Fz}^{2-}$ is in the same aqueous phase as the photosensitizer, so that it may also serve as an electron donor and quench the porphyrin excited state, or react with porphyrin cation radicals. Such reactions diminish the overall quantum yield of the photoreaction. When $\mathrm{MMP}^{+}$is left out from this mixture (system $\mathbf{L B}_{2}$ ), the reaction is only slightly inhibited $\left(\varphi_{0}=0.72 \%\right.$ ) compared to $\mathbf{L B}_{\mathbf{1}}$ (Fig. 1b), confirming that electron transfer from HEDTA ${ }^{3-}$ to $\mathrm{WST}^{-}$in $\mathbf{L B}_{\mathbf{1}}$ occurs within a single aqueous phase where no membrane needs to be crossed. The addition of triton X-100 to system $\mathbf{L B}_{\mathbf{1}}$ (i.e., system $\mathbf{L B}_{3}$ ) leads to an increase in the efficiency of the photoreaction, confirming that in $\mathbf{L A}_{\mathbf{3}}$ triton X-100 quenches the photoreaction solely by disrupting the liposomes.

Many photocatalytic systems are not active in the presence of dioxygen, which would pose a problem in a photocatalytic fuel production system based on water oxidation. Thus, the sensitivity of systems $\mathbf{L A}_{\mathbf{1}}$ and $\mathbf{L B}_{\mathbf{1}}$ to $\mathrm{O}_{2}$ was investigated. When system $\mathbf{L B}_{\mathbf{1}}$ was not deaerated (system $\mathbf{L B}_{\mathbf{4}}$ ) the presence of $\mathrm{O}_{2}$ did not significantly influence the rate of the photoreaction, nor did it cause any induction period common to this type of systems due to the initial competition between $\mathrm{WST}^{-}$and $\mathrm{O}_{2}$ to accept the first photoelectrons. ${ }^{4 g, 9}$ In contrast, when system

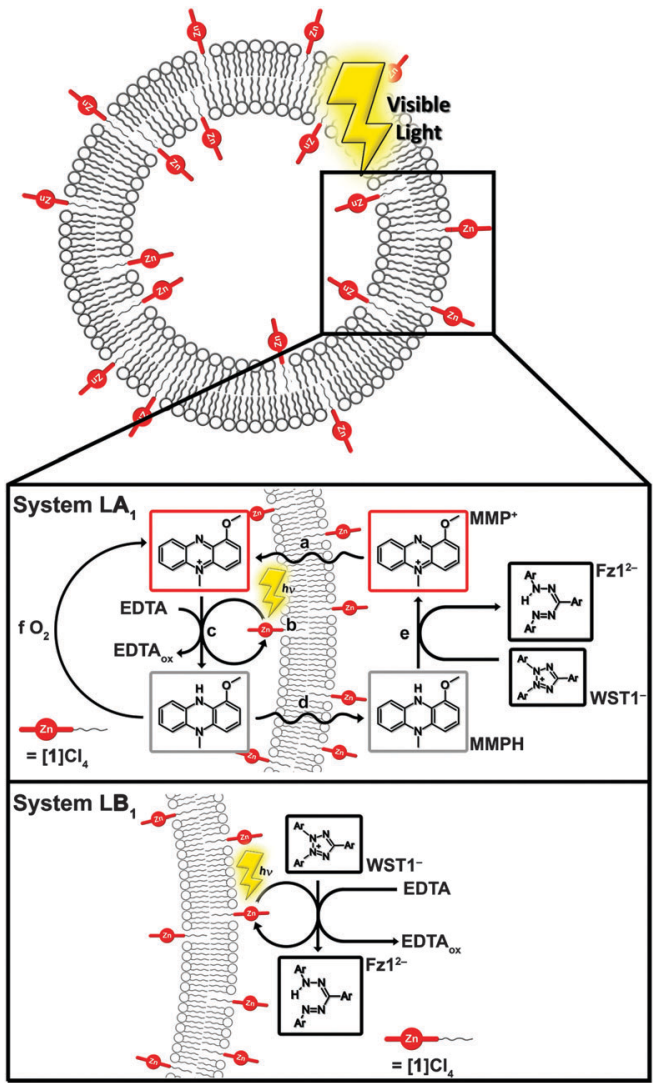

Fig. 2 Top: scheme of the photocatalytic reduction of $\mathrm{WST1}^{-}$by HEDTA $^{3-}$ with dye-functionalized liposomes. Middle: system LA $_{\mathbf{1}}$, with EDTA and WST1- on opposite sides of the membrane. (a) Passive diffusion of $\mathrm{MMP}^{+}$through the membrane into the liposome. (b) Absorption of light by $[1]^{4+}$. (c) Photocatalytic oxidation of EDTA (in the form of HEDTA ${ }^{3-}$ at $\mathrm{pH} 8$ ) and reduction of $\mathrm{MMP}^{+}$to $\mathrm{MMPH}$. (d) Passive diffusion of $\mathrm{MMPH}$ through the membrane. (e) Chemical (dark) reduction of $\mathrm{WST}^{-}$to $\mathrm{Fz1}^{2-}$ by $\mathrm{MMPH}$. (f) Undesired re-oxidation of $\mathrm{MMPH}$ to $\mathrm{MMP}^{+}$by molecular oxygen in system $\mathrm{LA}_{4}$. Bottom: system $\mathrm{LB}_{1}$, with HEDTA ${ }^{3-}$ and WST1- on the same side of the membrane. $\mathrm{MMP}^{+}$has been left out for clarity as it does not influence the system significantly.

$\mathbf{L}_{2} \mathbf{A}_{\mathbf{1}}$ was not deaerated (system $\mathbf{L}_{2} \mathbf{A}_{\mathbf{4}}$ ) complete bleaching of the Soret band of the porphyrin was observed and no $\mathrm{Fz}^{2-}$ was produced (Fig. S1b, ESI $\dagger$ ). The striking difference between systems $\mathbf{L}_{2} \mathbf{A}_{\mathbf{4}}$ and $\mathbf{L}_{2} \mathbf{A}_{\mathbf{1}}$ shows that one of the catalytic intermediates in $\mathbf{L} \mathbf{A}_{\mathbf{1}}$ and $\mathbf{L}_{\mathbf{2}} \mathbf{A}_{\mathbf{1}}$ is unstable in the presence of $\mathrm{O}_{2}$. We hypothesized that such an intermediate would be MMPH, as it plays a key role in the photocatalytic mechanism of system $\mathbf{L A}_{\mathbf{1}}$. In a control experiment, system $\mathbf{L A}_{\mathbf{1}}$ without $\mathrm{WST}^{-}$was irradiated in the absence of $\mathrm{O}_{2}$; in this reaction MMPH was produced, which could be detected by UV-vis spectroscopy (Fig. S2, ESI $\dagger$ left). After bubbling air through the solution the reaction was fully reversed: all $\mathrm{MMPH}$ was oxidized back to $\mathrm{MMP}^{+}$(Fig. S2, ESI $\dagger$ right). Thus, photocatalytic electron transfer through the membrane such as that observed in system $\mathbf{L}_{\mathbf{2}} \mathbf{A}_{\mathbf{1}}$ is sensitive to dioxygen because the reduced electron relay is rapidly oxidized by air.

Based on the observations described above the mechanism depicted in Fig. 2 (middle) is proposed for system $\mathbf{L} \mathbf{A}_{\mathbf{1}}$ and $\mathbf{L}_{2} \mathbf{A}_{\mathbf{1}}$. 
First $\mathrm{MMP}^{+}$, introduced in the bulk aqueous phase, diffuses through the membrane into the liposomes (a), ${ }^{7}$ where it is photoreduced with HEDTA $^{3-}$ (b). This reaction presumably occurs via reductive quenching of $*[1]^{4+}$ by HEDTA $^{3-}$ (high local concentration, $125 \mathrm{mM}$ ), followed by reduction of $\mathrm{MMP}^{+}$by $[1]^{3+}$. Oxidative quenching would be thermodynamically feasible (the redox potential of the $\mathrm{MMP}^{+} / \mathrm{MMPH}$ couple is $+0.06 \mathrm{~V} v s$. NHE), ${ }^{10}$ however kinetically unlikely due to the low concentration of $\mathrm{MMP}^{+}(42 \mu \mathrm{M})$. MMPH produced inside the liposomes can diffuse through the membrane in absence of $\mathrm{O}_{2}$, thus transporting the electrons and proton out of the liposome (d). Reduction of $\mathrm{WST}^{-}$by MMPH outside of the liposomes forms $\mathrm{Fz}^{2-}$ (e) and regenerates $\mathrm{MMP}^{+}$, as reported for the WST-1 cell counting assay. $\mathrm{MMP}^{+}$can then diffuse through the membrane again and turnover further. Since the amount of $\mathrm{Fz}^{2-}$ produced (e), monitored by UV-vis, exceeds the amount of $\mathrm{MMP}^{+}$present at the start of the reaction (Fig. 1a), $\mathrm{MMP}^{+}$must be concomitantly regenerated in the bulk aqueous phase, i.e., $\mathrm{MMP}^{+}$must be catalytic.

In summary, unlike previous studies in which electron transfer across lipid bilayers occurred concomitantly with the stoichiometric transport and occlusion of the electron acceptor into the membrane, directional electron transfer across a DPPC bilayer is unambiguously demonstrated here by combining a membrane-soluble electron relay, hydrophilic electron donors and acceptors situated inside and outside liposomes, respectively, and a membrane-bound photosensitizer. In this system the $\mathrm{MMP}^{+}$ electron relay truly acts catalytically in absence of oxygen, but under air it is oxidized and cannot fulfill its electron-transporting role. Under inert atmosphere, the actual transport of electrons across the membrane does not limit the reaction rate, as the initial quantum efficiency of the reaction through the membrane $\left(\mathbf{L A}_{\mathbf{1}}\right)$ is three times higher than when the donor and acceptor are both positioned on the same side of the membrane $\left(\mathbf{L B}_{\mathbf{1}}\right)$. Keeping the photoreduced electron acceptor physically separated from the electron donor allows for preventing product inhibition, i.e., quenching of the porphyrin excited states by $\mathrm{Fz}^{2-}$, which would otherwise diminish the quantum efficiency of the photoreduction. Finding a membrane-soluble electron relay that is stable in the presence of dioxygen will be required for the future development of supramolecular photocatalytic systems in which water oxidation and proton reduction catalysts are combined.

\section{Notes and references}

1 (a) N. Ikuta, S.-y. Takizawa and S. Murata, Photochem. Photobiol. Sci., 2014, 13, 691-702; (b) M. Hansen, F. Li, L. Sun and B. König, Chem. Sci., 2014, 5, 2683-2687; (c) S. Troppmann and B. König, Chem. Eur. J., 2014, 20, 14570-14574.

2 (a) S. Bhosale, A. L. Sisson, P. Talukdar, A. Fürstenberg, N. Banerji, E. Vauthey, G. Bollot, J. Mareda, C. Röger, F. Würthner, N. Sakai and S. Matile, Science, 2006, 313, 84-86; (b) C. J. Grubb, D. J. ColeHamilton and M. K. Whittlesey, J. Chem. Soc., Faraday Trans., 1996, 92, 5005-5012; (c) Z.-G. Zhao and G. Tollin, Photochem. Photobiol., 1992, 55, 611-619; (d) M. M. A. Kelson, R. S. Bhosale, K. Ohkubo, L. A. Jones, S. V. Bhosale, A. Gupta, S. Fukuzumi and S. V. Bhosale, Dyes Pigm., 2015, 120, 340-346; (e) G. Steinberg-Yfrach, P. A. Liddell, S.-C. Hung, A. L. Moore, D. Gust and T. A. Moore, Nature, 1997, 385, 239-241; $(f)$ G. Steinberg-Yfrach, J. L. Rigaud, E. N. Durantini, A. L. Moore, D. Gust and T. A. Moore, Nature, 1998, 392, 479-482.

3 (a) R. F. Khairutdinov and J. K. Hurst, Nature, 1999, 402, 509-511; (b) S. V. Lymar, R. F. Khairutdinov, V. A. Soldatenkova and J. K. Hurst, J. Phys. Chem. B, 1998, 102, 2811-2819.

4 (a) K. Watanabe, K. Moriya, T. Kouyama, A. Onoda, T. Minatani, S.-Y. Takizawa and S. Murata, J. Photochem. Photobiol., A, 2011, 221, 113-122; (b) R. Sasaki, Y. Nako and S. Murata, Tetrahedron, 2009, 65, 7364-7371; (c) T. Mizushima, A. Yoshida, A. Harada, Y. Yoneda, T. Minatani and S. Murata, Org. Biomol. Chem., 2006, 4, 4336-4344; (d) A. Yoshida, A. Harada, T. Mizushima and S. Murata, Chem. Lett., 2003, 32, 68-69; (e) J. N. Robinson and D. J. Colehamilton, Chem. Soc. Rev., 1991, 20, 49-94; $(f)$ S. V. Lymar, V. N. Parmon and K. I. Zamaraev, Top. Curr. Chem., 1991, 159, 1-65; $(g)$ W. E. Ford, J. W. Otvos and M. Calvin, Proc. Natl. Acad. Sci. U. S. A., 1979, 76, 3590-3593; (h) W. E. Ford, J. W. Otvos and M. Calvin, Nature, 1978, 274, 507-508.

5 (a) L. Hammarström and M. Almgren, J. Phys. Chem., 1995, 99, 11959-11966; (b) L. Hammarström, H. Berglund and M. Almgren, J. Phys. Chem., 1994, 98, 9588-9593; (c) E. R. Kuhn and J. K. Hurst, J. Phys. Chem., 1993, 97, 1712-1721; (d) L. Hammarström, M. Almgren, J. Lind, G. Merenyi, T. Norrby and B. Akermark, J. Phys. Chem., 1993, 97, 10083-10091; (e) L. Hammarström and M. Almgren, Proc. -Indian Acad. Sci., Chem. Sci., 1993, 105, 539-554; $(f)$ S. V. Lymar and J. K. Hurst, J. Am. Chem. Soc., 1992, 114, 9498-9503; $(g)$ L. Hammarström, M. Almgren and T. Norrby, J. Phys. Chem., 1992, 96, 5017-5024; (h) B. C. Patterson, D. H. Thompson and J. K. Hurst, J. Am. Chem. Soc., 1988, 110, 3656-3657; (i) J. K. Hurst and D. H. P. Thompson, J. Membr. Sci., 1986, 28, 3-29; ( $j$ ) L. Y. C. Lee, J. K. Hurst, M. Politi, K. Kurihara and J. H. Fendler, J. Am. Chem. Soc., 1983, 105, 370-373.

6 L. Zhu, R. F. Khairutdinov, J. L. Cape and J. K. Hurst, J. Am. Chem. Soc., 2006, 128, 825-835.

7 M. Ishiyama, M. Shiga, K. Sasamoto, M. Mizoguchi and P. G. He, Chem. Pharm. Bull., 1993, 41, 1118-1122.

8 K. Kalyanasundaram and M. Neumann-Spallart, J. Phys. Chem., 1982, 86, 5163-5169.

9 B. Limburg, G. Laisné, E. Bouwman and S. Bonnet, Chem. - Eur. J., 2014, 20, 8965-8972.

10 R. Hisada and T. Yagi, J. Biochem., 1977, 82, 1469-1473. 